\title{
In this issue
}

\section{Ironing out the molecular details of aldosterone production}

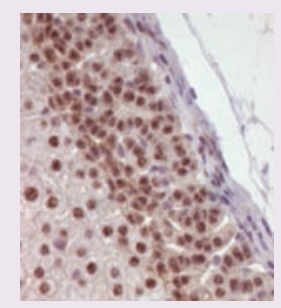

Recent data have indicated that aldosterone is an early-onset pathogenic stimulus of heart failure. Aldosterone is produced by zona glomerulosa cells in the adrenal cortex in a $\mathrm{Ca}^{2+}$-dependent manner. Low-voltage activated T-type $\mathrm{Ca}^{2+}$ channels are opened by depolarization and maintain the elevated concentration of intracellular $\mathrm{Ca}^{2+}$ that is required to sustain the production of aldosterone. $\mathrm{Ca}^{2+} /$ calmodulin-dependent protein kinase II (CaMKII) is known to have a regulatory role in this process, but its sites of action had not been determined. Yao and colleagues now show that inactive CaMKII binds the intracellular loop connecting transmembrane domains II and III of the $\alpha_{1 \mathrm{H}}$ T-type $\mathrm{Ca}^{2+}$ channel (pages 2403-2412). Activation of CaMKII was found to induce CaMKII-mediated phosphorylation of serine residue 1198 of the $\alpha_{1 \mathrm{H}}$ channel, which in turn led to the release of CaMKII. Importantly, a CaMKII-dependent increase in phosphorylation of serine residue 1198 was observed in rats infused with angiotensin II, which is a physiological regulator of aldosterone production. This study provides new insight into the molecular mechanisms regulating the $\alpha_{1 \mathrm{H}}$ T-type $\mathrm{Ca}^{2+}$ channel and might present researchers with new therapeutic approaches to regulating aldosterone production.

\section{NF1: think outside the GRD}

Neurofibromatosis type I (NF1) is a monogenetic disorder that mostly causes abnormalities in neural crest-derived tissues, the most severe of which are tumors. It is caused by mutation of the NF1 gene, which encodes neurofibromin. The only currently wellcharacterized domain of neurofibromin is one with Ras GTPase-activating protein (GAP) activity. Defects in this GAP-related domain (GRD) have been linked to the development of NF1-related tumors, and clinical trials to target this defect are currently underway. However, other studies indicate that mutations in other regions of neurofibromin can lead to NF1. So, Ismat and colleagues used a mouse model of NF1 to examine the relative roles of the GRD and the other regions of neurofibromin in disease (pages 2378-2384). Expression of the human NF1 GRD in $\mathrm{Nf1}^{-/-}$mice was shown to rescue the embryos from mid-gestation lethality due to cardiovascular defects. However, neural crest-derived tissues in the $N f 1^{-/-}$mice expressing the human NF1 GRD grew abnormally and resembled the neural crest-derived tumor-like lesions observed in mice in which Nf1 is inactivated only in neural crest tissues. These observations indicate that loss of Ras regulatory activity in NFI is unlikely to be the only cause of the abnormalities in neural crest-derived tissues. This has important implications for the ongoing clinical trials that are aimed at targeting Ras activity to treat NF1related tumors and suggest that other functions for neurofibromin remain to be discovered.

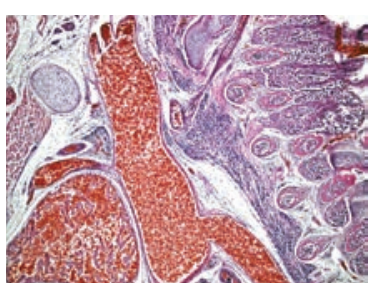

\section{PTEN blocks the way for Tregs}

Naturally occurring $\mathrm{CD} 4^{+} \mathrm{CD} 25^{+}$Tregs function to prevent self-reactive T cells from destroying self-tissues. But harnessing the therapeutic potential of Tregs is hampered because, unlike nonregulatory $T$ cells, they do not proliferate in vitro when stimulated through their TCR, and, unlike activated $\mathrm{CD} 4^{+} \mathrm{CD} 25^{+}$effector $\mathrm{T}$ cells, they do not undergo cell division in response to IL-2. Walsh and colleagues had previously observed that signaling downstream of the receptor for IL-2 was distinct in Tregs and nonregulatory $\mathrm{T}$ cells. In particular, increased expression of phosphatase and tensin homolog (PTEN), which is a negative regulator of PI3K signaling pathways, was detected in Tregs. In this issue, the authors now report that in mice, $\mathrm{T}$ cell-specific deletion of Pten results in the development of normal numbers of Tregs but that PTEN-deficient Tregs proliferate when stimulated with IL-2 ex vivo and undergo increased homeostatic proliferation in vivo (pages 2521-2531). Importantly, PTEN-deficient Tregs retained their suppressive function in vitro and in vivo, indicating that loss of PTEN only affects the IL-2-hypoproliferative phenotype of these cells. This study identifies one of the molecular regulators of the IL-2-hypoproliferative phenotype of Tregs and could provide researchers with a way to overcome one of the biggest obstacles to harnessing their therapeutic potential.

\section{Fox01's dual personality}

Insulin resistance is a central feature of metabolic diseases. However, in the liver, increased glucose production (due to insulin resistance) often coexists with increased triglyceride synthesis and decreased fatty acid oxidation, which are signs of increased insulin sensitivity. One explanation for this paradox is that insulin regulates glucose and lipid metabolism through distinct pathways. But now, Matsumoto and colleagues show that forkhead box O1 (FoxO1), which is known to induce hepatic glucose production, also promotes hepatic insulin sensitivity (pages 2464-2472). Expression of a constitutively nuclear form of FoxO1 in mouse liver was found to increase triglyceride synthesis and decrease fatty acid oxidation. This was associated with increased phosphorylation of Akt, which was not mediated by FoxO1 functioning as a transcription factor. Instead, FoxO1 was shown to decrease the expression of tribble 3 (Trb3), which inhibits Akt phosphorylation. This demonstration that FoxO1 regulates the pathway controlling lipid metabolism, together with its known role in the pathway that regulates glucose production, indicates that a single protein influences both glucose and lipid metabolism. This provides a new explanation for the dual symptoms of hepatic insulin resistance and sensitivity often seen in individuals with metabolic diseases.

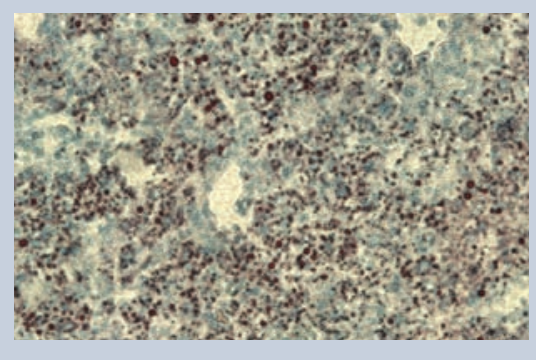

